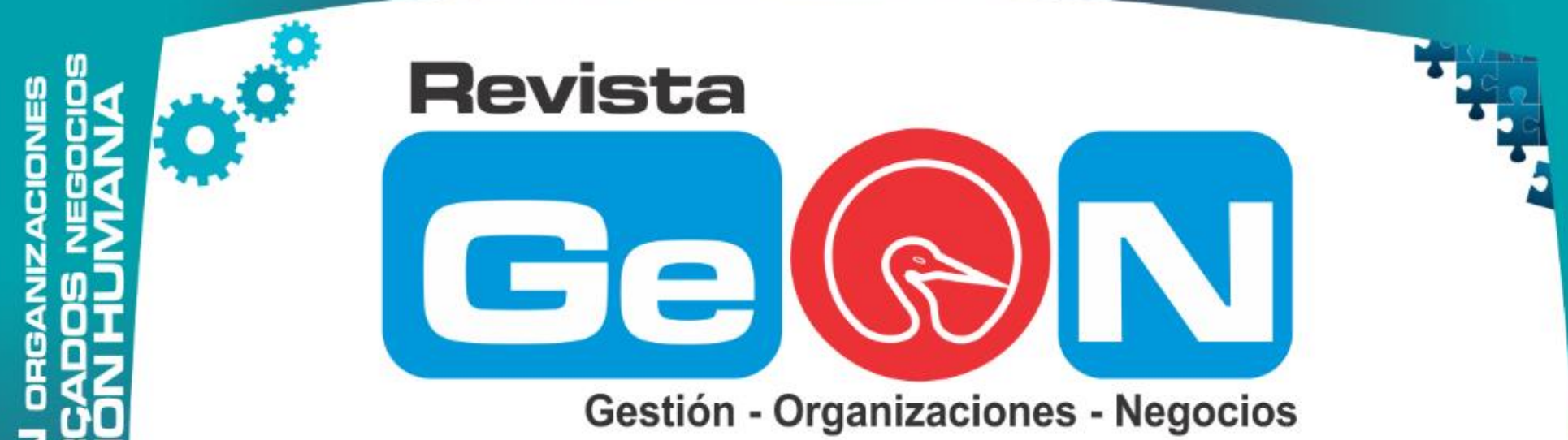

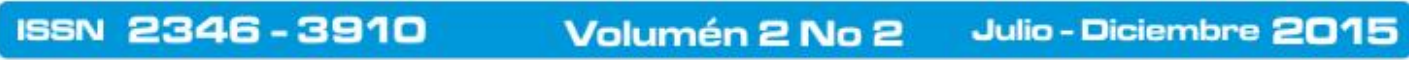

Revista Electrónica de la Facultad de Ciencias Económicas de la Universidad de los Llanos

1. 3 II

440

VILLAVICENCIO - COLOMBIA

$>2$

64

2

2 平品

70

ব)

展

o

ii III

0 U

ใด

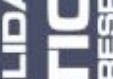

居的

임

Iiin in

照

只

造

iil 2

if in

4 吕

2

400

付 iI
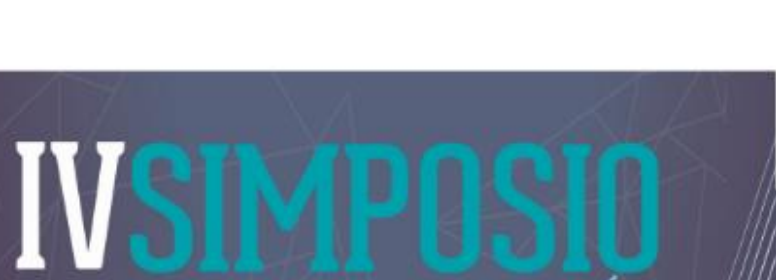

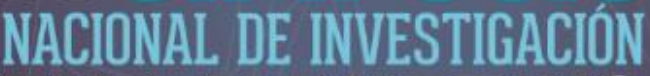

EN MARKETING
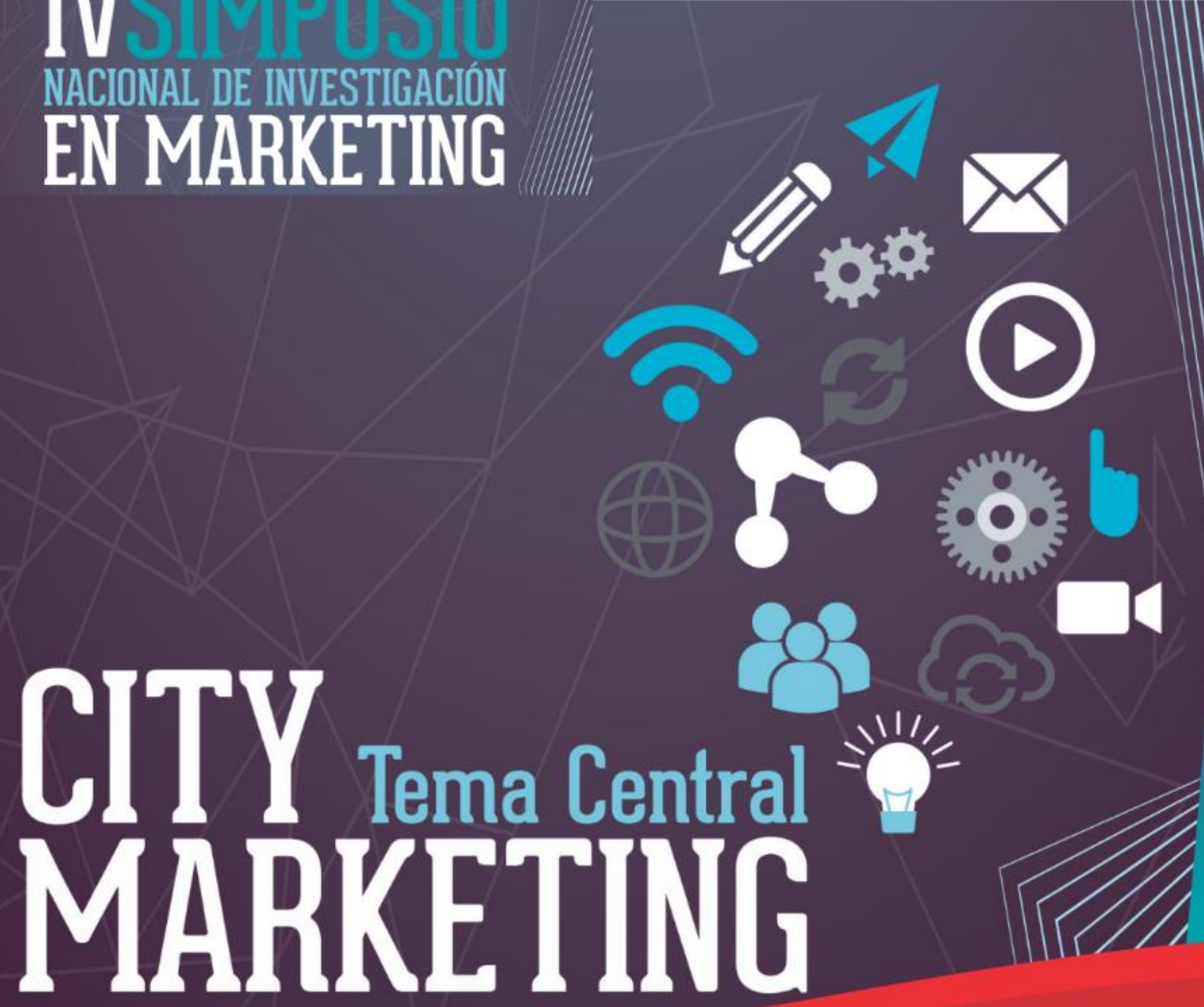

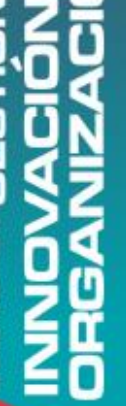
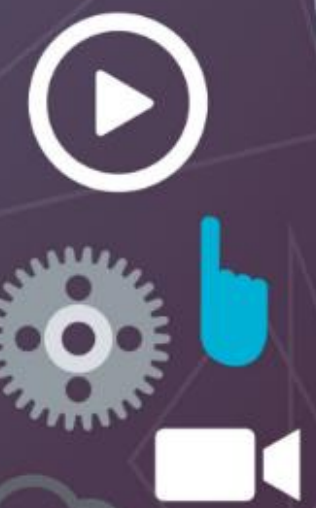


\section{RESPONSABILIDAD SOCIAL Y SOSTENIBILIDAD}

El marketing y la responsabilidad social y empresarial, desarrollado con habitantes de la vereda Candelaria, municipio de Ráquira Boyacá - Ángel Ávila

Ángel Leonardo Ávila Castañeda

Especialista en Comportamiento del Consumidor y (C) Magister en Dirección de Marketing

Angel.avila@uniagustiniana.edu.co @anleaoav

\section{Resumen}

En el marco de la aplicación del marketing social como herramienta que influye en el comportamiento voluntario de la audiencia objetivo, con el fin de mejorar su bienestar personal y de la comunidad, se documenta el proceso de investigación realizado por los (as) estudiantes del semillero de mercadeo "Marketeros en Acción" donde se desarrolló un diagnóstico, ejecución de capacitaciones y creación de un plan de marketing para el desarrollo de un proyecto viable sostenible con grupos de emprendedores y comerciantes de la verada la Candelaria del Municipio de Ráquira, Departamento de Boyacá.

\section{Palabras clave}

Estrategias de marketing; desarrollo sostenible; microempresarios; capacitaciones.
Abstract
In the framework of the implementation of social marketing as a tool to influence the voluntary behavior of target audiences in order to improve their personal welfare and community, the research process conducted by the (as) students documented seed marketing " marketers in Action" where a diagnosis, implementation of training and creating a marketing plan for the development of a sustainable viable project with groups of entrepreneurs and traders verada Candelaria Township Ráquira, Boyacá Department developed.

\section{Keywords}

Marketing strategies, sustainable development, micro entrepreneurs, and trainings.

\section{Introducción}

Cuando hablamos de los conceptos del marketing y sus estrategias por lo general lo asociamos con grandes marcas $y$ altos presupuestos; la Asociación Americana de Marketing (American Marketing Association) ${ }^{10}$ lo define "Marketing es una función de la organización y un conjunto de procesos dirigidos a crear, comunicar y distribuir valor a los clientes y a dirigir las relaciones con los clientes de forma que beneficie a la organización y sus públicos de interés".

Las grandes empresas aplican para su desarrollo y posicionamiento estos conceptos, implementando dentro de sus estructuras, departamentos de mercadeo para lograr los fines corporativos y de competitividad en el mercado; acciones que hoy en día microempresarios y personas emprendedoras buscan adoptar en sus negocios e ideas desarrollándolas empíricamente o con fundamentos muy básicos buscando el apoyo de profesionales de mercadeo para mejorar sus conocimientos conducentes a la realización de estrategias para sus negocios.

En este artículo incorporamos el concepto de Marketing Social tal como lo define Alan Andreasen (2002), quien lo establece como "la aplicación de las técnicas del marketing comercial para el análisis, planteamiento, ejecución y evaluación de programas diseñados para influir en el comportamiento voluntario de

\footnotetext{
${ }^{10}$ En agosto de 2004 la AMA publica una nueva definición de marketing aprobada por la American Marketing Association Board of Directors.
} 
la audiencia objetivo en orden a mejorar su bienestar personal y el de la sociedad".

Encontraremos los resultados de investigación y participación por parte de los alumnos (as) del programa de mercadeo vinculado al grupo de responsabilidad social tal como se define en la Uniagustiniana ${ }^{11}$, donde se desarrolló, un diagnóstico, capacitaciones e implementación de estrategias con grupos de emprendedores y comerciantes de la vereda.

\section{Desarrollo}

El trabajo de investigación e implementación de acciones tiene dos actividades fundamentales:

La primera actividad surge gracias a la invitación por parte del programa de Arquitectura de la Universitaria Agustiniana donde nos hacen participes del Taller Asociado de La Candelaria cuyo objetivo es la integración de diferentes programas como son ( Arquitectura, Gastronomía Mercadeo); en donde se busca elaborar un plan de acción conjunto con la comunidad, el programa de Mercadeo de la Universitaria Uniagustiniana, estableció como objetivo se estableció en brindar a los comerciantes los conceptos teórico - prácticos, de mercadeo y ventas, aplicando las herramientas necesarias para dirigir, controlar, implementar y evaluar las estrategias que les permitan ser más competitivos en las exigencias del mercado actual, e implementar estrategias de mercadeo de forma conjunta con un estudio de caso, para lograr este objetivo, las acciones se desarrollaron en varias fases.

La primera fase se desarrolló una investigación de tipo exploratorio descriptivo, a

${ }^{11}$ La Universitaria Uniagustiniana establece "la unidad de Proyección Social que dinamiza la materialización en la comunidad universitaria del componente social.

Las acciones socialmente responsables de UNIAGUSTINIANA, involucran procesos académicos, administrativos y de investigación, que bajo criterios de necesidad y pertinencia global, contribuyen a la justicia social y las buenas prácticas institucionales" través de un enfoque mixto (cualitativo y cuantitativo) a partir de la cual se formuló un diagnóstico, que permitió conocer e identificar las necesidades de la población de la vereda la Candelaria municipio de Ráquira, esta investigación se realizó en tres etapas:

Entrevistas realizadas al personero municipal, los comerciantes y pobladores de la zona.

Análisis del plan de desarrollo "Porque Ráquira somos todos 2012-2015 (Alcalde Municipal Jose Hernan Sierra Buitrago, 2012)"

Aplicar al sector del comercio y servicios hoteleros, de la vereda y el municipio.

La recolección de datos se realizó directamente con los comerciantes obteniendo una población muestral de cinco comerciantes de la vereda de la candelaria y cuarenta y dos (42) comerciantes del municipio de Ráquira.

La segunda fase se enfocó en él desarrollo de las capacitaciones logrando una participación de 16 comerciantes de la Vereda de la Candelaria y el municipio de Ráquira logrando aceptación frente a los temas y la metodología empleada, consolidándose en una encuesta de satisfacción realizada a todos los participantes.

Para la tercera fase de participación del programa de Mercadeo en el Taller Asociado de la Candelaria se estableció el objetivo de desarrollar todo un plan de acción con una familia específica, gracias a la gestión del programa de arquitectura en el desarrollo de la estructura de una panadería, el programa de mercadeo y su grupo de semilleros enfoca su plan de acción en el desarrollo de estrategias para la comercialización y posicionamiento de la panadería llamada "El Pan Campesino de Mama Teolita" estas actividades se desarrollaron en el acompañamiento de la distribución de los productos de la panadería, con el fin de realizar un diagnóstico de la zona y una retroalimentación al señor Alexander Rodríguez en cuanto a las estrategias de ventas. Otra de las acciones de esta fase es el desarrollo de un material publicitario (pendón y volantes) con el 
fin de facilitar la comunicación y caracterización de la panadería y de un manual para capacitación con conceptos claves para el desarrollo de un plan de marketing con actividades de desarrollo sostenible y economía solidaria.

Los resultados de las diferentes fases de capacitación logrando la presentación de las siguientes discusiones:

Para el desarrollo de la primera fase se desarrolló un diagnóstico para identificar las necesidades de capacitación de los comerciantes, dentro de los resultados más relevantes encontramos:

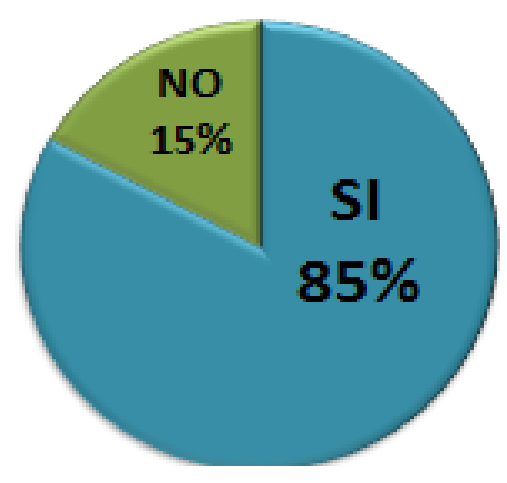

Figura 1. Pregunta ¿Está usted dispuesto en recibir capacitaciones con temas de mercadeo y

Ventas?

Se logró una aceptación del $85 \%$ de la población encuestada, en recibir capacitaciones con temas de mercadeo y ventas ya que el $60 \%$ no conocía de conceptos claves en el desarrollo de estrategias de los mismos temas.

Con el diagnostico se identificaron cuatro temas, desarrollados para la capacitación, los cuales fueron:

Donde se tuvo a bien exponer los siguientes temas:

\section{Contenido módulo conceptos de mercadeo}

(Terminología aplicada en Mercadeo Conceptos generales de mercadeo - Aplicación de concepto al desarrollo de sus actividades, (Casos prácticos)

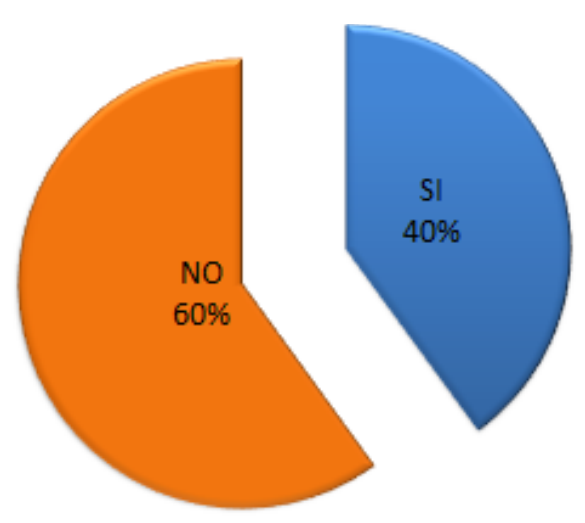

Figura 2. Pregunta. ¿Conoce acerca de estrategias de mercadeo y ventas?

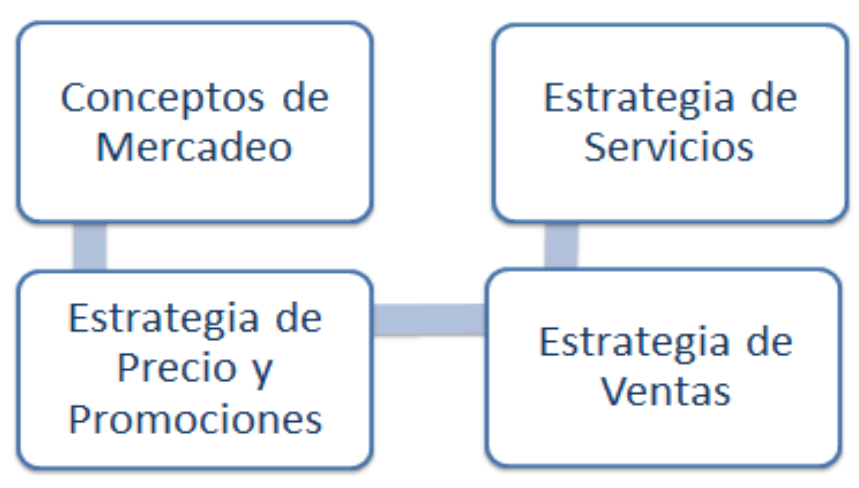

Figura 3. Temas desarrollados en las capacitaciones del municipio de Ráquira

\section{Contenido módulo estrategia de precio y promociones}

Estrategia de Precios. - Comparación con la competencia. - Control de precios. - Márgenes de comercialización de los canales de venta. Descripción de las estrategias de promoción Tipos de estrategias (evento de lanzamiento, publicidad, merchandising, material pop, relaciones públicas, venta directa ) - Medios de comunicación

\section{Contenido módulo estrategia de ventas}


Descripción de las estrategias de venta Estrategia de venta directa - Estrategia de venta Online - Estrategia de venta automatizada Descripción de la estructura de ventas Políticas de venta - Técnicas de venta - Proceso de la venta (descripción y flujo grama) Programa de ventas - Descripción perfil de vendedor.

\section{Contenido módulo estrategia de servicios}

Descripción de la estrategia de servicios Descripción del ciclo del servicio Triangulo de servicio - Las garantías, de las devoluciones, los descuentos y de postventa La estrategia de contactos con los clientes; telefónico o personal (in bound - out bound) - Call center \& redes sociales.

Se expuso de acuerdo con Kotler \& Armstrong (2008), que el mercadeo es el proceso por el cual las empresas crean valor para sus clientes, a través de actividades humanas dirigidas a facilitar intercambios que les permiten construir fuertes relaciones con ellos, con el propósito de obtener a cambio valor procedente de dichos clientes.

Resultó de vital importancia capacitar a esta comunidad en el tema de identificación de necesidades, para ello se tomó como base a la teoría sobre la motivación humana de Maslow $(1943)^{12}$ quien expone, que las necesidades se clasifican en orden de satisfacción iniciando en las fisiológicas, las de seguridad, necesidades sociales, de estima y por último la necesidad de auto-realización. Otro de los conceptos claves que se trató en estas capacitaciones a empresarios fue la importancia de la segmentación como base para ofertar sus productos artesanales efectivamente.

\footnotetext{
${ }^{12}$ Teoría Psicológica Propuesta por Abraham Maslow en su obra Una Teoría sobre la Motivación Humana, formulando una jerarquía de necesidades humanas y defiende que conforme se satisfacen las necesidades más básicas (parte inferior de la pirámide), los seres humanos desarrollan necesidades y deseos más elevados (parte superior de la pirámide).
}

El concepto de marketing mix, que expone Kotler \& Keller (2012) sobre las 4p’s (Producto, Precio, Plaza, Promoción) fue una de las bases fundamentales de la capacitación, desglosando cada una en los módulos propuestos; brindando estos conceptos como herramientas estratégicas para el incremento de sus ventas y el posicionamiento de los productos autóctonos de la región.

Las capacitaciones se desarrollaron con 16 participantes integrados por comerciantes del municipio de Ráquira y la Vereda de la Candelaria.

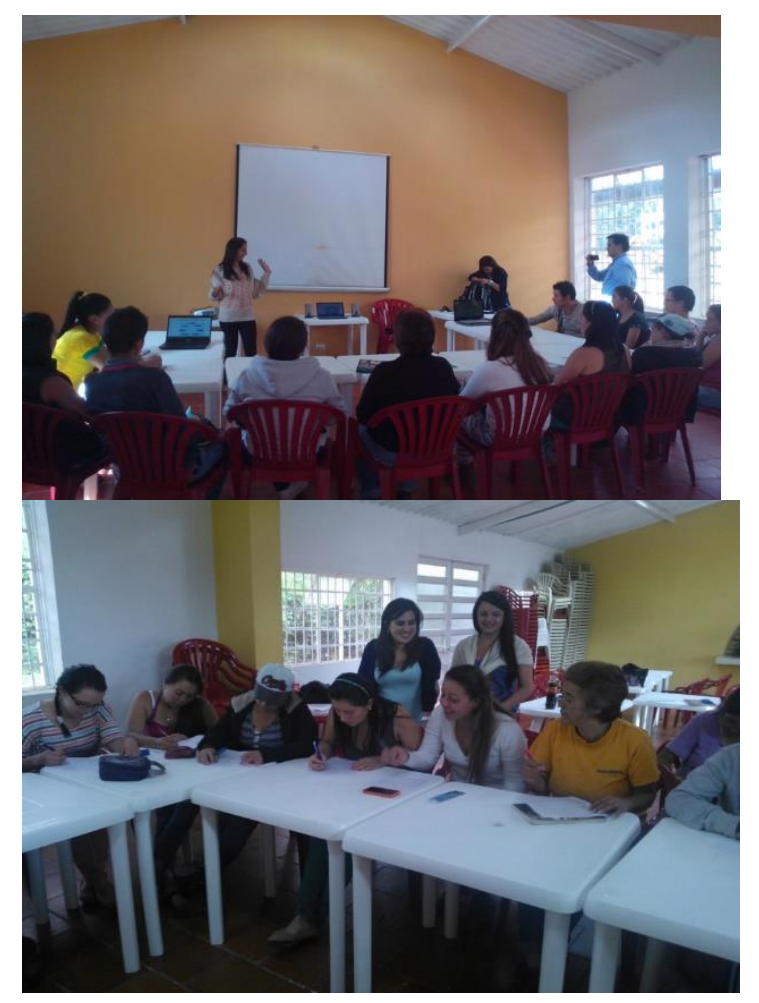

Imágenes 1 y 2. Fotos capacitación municipio de Ráquira

Al terminar la capacitación, se realizó la evaluación de satisfacción de la capacitación, donde tenemos los siguientes resultados: 


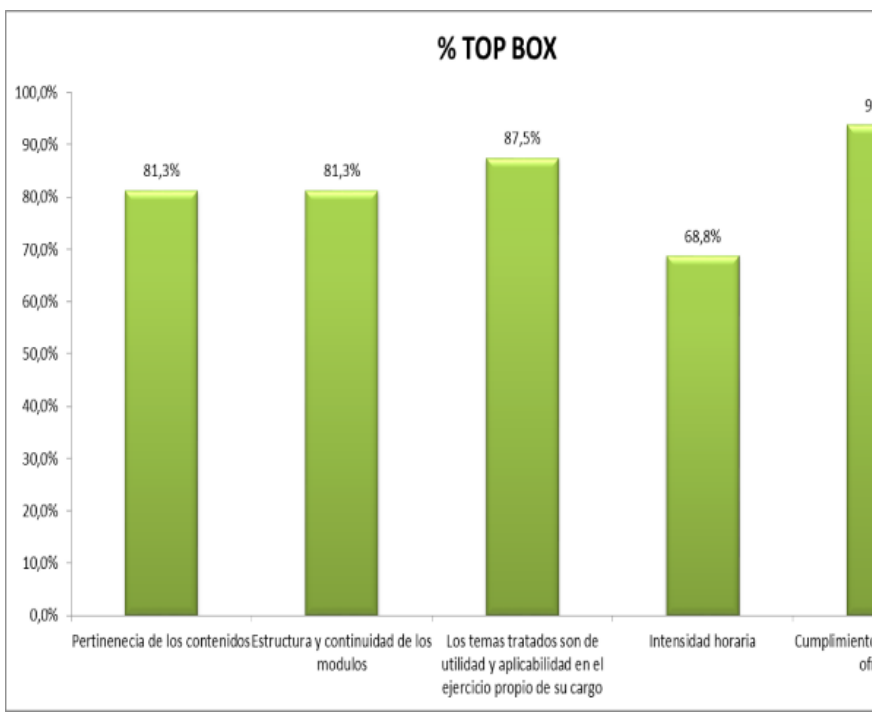

Figura 4. Encuesta de satisfacción capacitación de mercadeo Municipio de Ráquira

Se logró una evaluación positiva en general, midiendo variables como la pertinencia de los contenidos con una calificación y estructura de continuidad de los módulos con 81.3 puntos, los temas tratados son de utilidad y aplicabilidad en el ejercicio propio de su cargo con 87.5 puntos, 68.8 puntos de calificación en intensidad horaria, siendo la calificación más baja, dado que las horas de trabajo fueron de 4 horas única jornada, siendo un tiempo corto para dicha actividad esto justificado por la disposición de los participantes y del tiempo otorgado en la fase que se implementó dicha labor. Y por último con 91,8 puntos se calificó el cumplimiento del cronograma ofrecido.

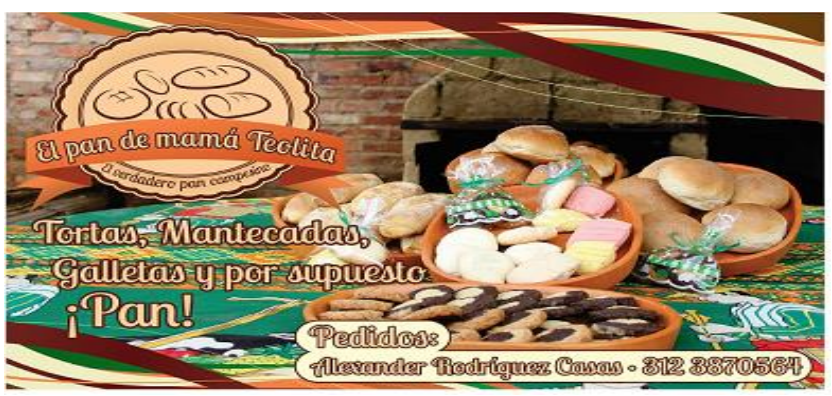

Imagen 3. Arte Pendón y Volantes Panadería "El Pan Campesino de Mama Teolita"

En la tercera fase se desarrolló el plan de acción para la comercialización y posicionamiento de la panadería "El Pan Campesino de Mama Teolita" de la Familia
Rodríguez, se desarrolló el material pop ( pendón y volantes ) entendiendo este concepto según Chong (2007), como el material publicitario o promocional utilizado para la decoración de tiendas por departamento que busca dar a conocer las diversas cualidades de un producto, obteniendo como pieza final los siguientes resultados.

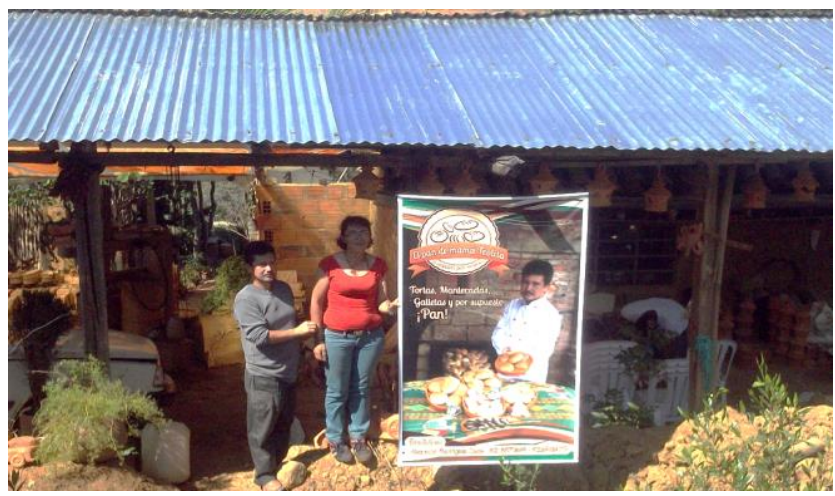

Imagen 4. Ubicación Pendón Panadería

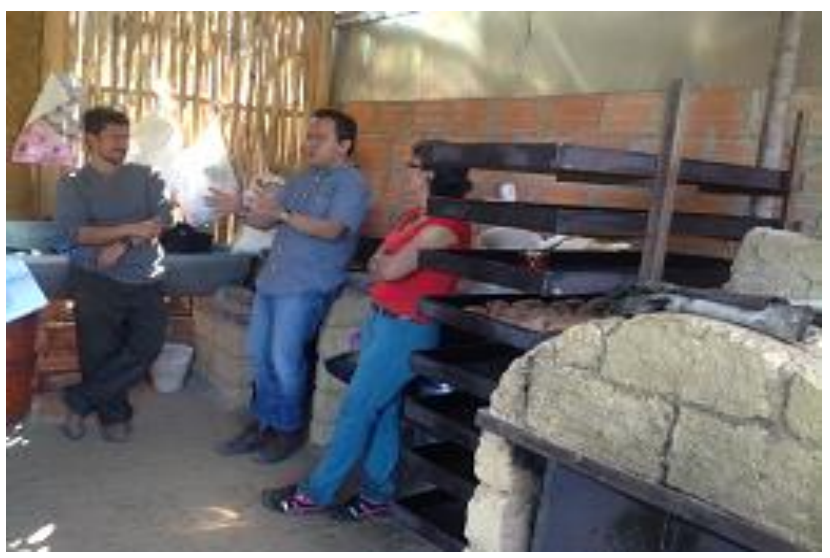

Imagen 5. Socialización Manual Plan de Marketing Familia Rodríguez

Asi mismo se desarrolló la capacitacion y socialización del manual con los pasos para aplicar un plan de mercadeo y de los conceptos de desarrollo sostenible y economia solidaria, entendiendo dichos conceptos como: La economía solidaria es un sistema socioeconómico , cultural y ambiental conformado por el conjunto de fuerzas sociales organizadas en formas asociativas identificadas por prácticas autogestionarias solidarias, democráticas y humanistas, sin ánimo de lucro 
para el desarrollo integral del ser humano como sujeto, actor y fin de la economía.(Ley 454/98 art. 2) y se entiende por desarrollo sostenible el que conduzca al crecimiento económico, a la elevación de la calidad de la vida y al bienestar social, sin agotar la base de recursos naturales renovables en que se sustenta, ni deteriorar el medio ambiente o el derecho de las generaciones futuras a utilizarlo para la satisfacción de sus propias necesidades. (Ley 99/93 título II Art. 3).

\section{Conclusiones}

Con la investigación encontramos los siguientes hallazgos:

Hoy en día el mercado es exigente, no importa si son marcas reconocidas o productos de grandes o pequeñas empresas, siempre buscan lo mejor en cuanto a calidad, servicio y precio, las empresas deben tener la suficiente experticia para poder generar las estrategias necesarias para lograr mantener sus ingresos de forma rentable, y es así como los comerciantes del municipio de Ráquira han entendido esa necesidad de estar capacitados y preparados para generar esas estrategias de marketing que logren aumentar sus ventas o incursionar en nuevos mercados.

El desarrollo de estas actividades nos permitió, tanto a docentes y alumnos, entender las dimensiones que pueden llegar a cubrir las estrategias del marketing, como así mismo conocer nuestro nivel de conocimiento ya que nos exige prepararnos para lograr trasmitir los conceptos y más aún lograr su aplicación en cada una de esas ideas o crecimiento de los negocios.

Los resultados de estas actividades han sido positivas y enriquecedoras ya que ha permitido ver el crecimiento tanto económico y de estabilidad emocional de la familia Rodríguez como de los demás participantes de las capacitaciones y a su vez el fortalecimiento de futuros y excelentes profesionales de mercadeo de la Universitaria Uniagustiniana.

\section{Referencias}

Alan R. Andreasen (2002) "Marketing Social en el Cambio Social del mercado". Diario de Políticas Públicas y Marketing: Primavera 2002, Vol. 21, No. 1, pp. 3-13.

Municipio de Ráquira (2012) "Plan de Desarrollo: Porque Ráquira Somos Todos 20122015".

Kotler \& Armstrong (2008) "Fundamentos de Marketing”. 8va ed. México. Pearson Educación.

Kotler \& Keller (2012)" Dirección de Marketing”, 14e.Mexico. Pearson Educación.

Chong.J (2007). "Promoción de ventas". Buenos Aires. Granica S.A

"Marco conceptual de la regulación de la economía solidaria". Ley 454.Articulo 2 (1998)

"Ley del medio ambiente.99.Titulo 2 Art 33" (1993) 